\title{
Estrogen modulation of pain perception with a novel $17 \beta$-estradiol pretreatment regime in ovariectomized rats
}

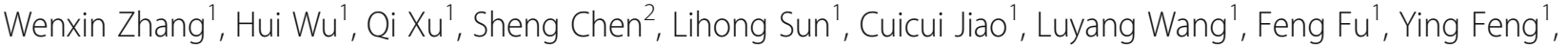 \\ Xiaowei Qian ${ }^{1}$ and Xinzhong Chen ${ }^{1 *}$
}

\begin{abstract}
Estrogen plays substantial roles in pain modulation; however, studies concerning sex hormones and nociception often yield confusing results. The discrepancy could be a result of lack of consensus to regard estrogen as a variable when working with animal models; thus, the influence of hormones' fluctuations on nociception has continually been neglected. In the present study, we designed a novel hormone substitution model to aid us to evaluate the effects of estrogen's long-term alterations on ovariectomy (OVX)-induced mechanical hyperalgesia and the expression of estrogen receptors(ERs). OVX rats were implanted with slow-release estrogen pellets at differently arranged time points and doses, such that a gradual elevation or decrease of serum estrogen levels following a relatively stable period of estrogen replacement was achieved in rats. Our results demonstrated that gradual estrogen depletion rather than elevation following the stable period of estrogen substitution in OVX rats alleviated OVX-induced mechanical hyperalgesia in a dose-independent manner, and the opposite estrogen increase or decrease paradigms differently regulate the expression of spinal ERs. Specifically, in rats rendered to continuously increased serum estrogen, the early phase estrogen-induced anti-nociception effect in OVX rats was eliminated, which was accompanied by an over-activation of ERa and a strong depression of ERB, while in the OVX rats subject to gradual decrease of estrogen replacement, both ERa and ERß increased modestly compared with the OVX group. Thus, the present study demonstrated that estrogen increase or decrease modulate nociception differently through change of spinal ERs.
\end{abstract}

Keywords: Estrogen, Nociception, Hyperalgesia, Estrogen receptors

\section{Introduction}

It is commonly recognized that females manifest different pain sensitivity under physiological and pathological conditions compared with males, and numerous chronic pain syndromes tend to aggravate during the premenopausal and ovulation periods. Estrogen is perceived to be a fundamental contributor to the sex-based disparities in nociceptive responses. However, previous studies yielded confusing results and both pro- and antinociceptive effects of estrogen have been observed. A tempting explanation for these contradictions might be that most of the studies investigated only the causal relationship

\footnotetext{
*Correspondence: chenxinz@zju.edu.cn

'Department of Anesthesia, Women's Hospital, Zhejiang University School of Medicine, Xueshi Road 1, Hangzhou 310006, China

Full list of author information is available at the end of the article
}

between the presence or absence of estrogen and the consequent behavioral or biological responses. However, the continuous increase or decrease of estrogen that was also important in pain modulation has been neglected.

The ever-changing characteristic of estrogen, as is manifested by its fluctuation during the menstrual cycle and peri-menopause periods, makes it extremely difficult to reveal the mechanisms underlying its pain modulatory effects during diverse physiological and pathological conditions. The majority of animal studies endeavored to investigate estrogen's effects on nociception had adopted the gonadectomy and hormone replacement modalities, and by this means, they simplified the essential clinical settings into a paradigm in which only the presence or absence of estrogen is considered, and thus failed to treat estrogen as a variable factor. Consequently, the related

(c) The Author(s). 2020 Open Access This article is distributed under the terms of the Creative Commons Attribution 4.0 International License (http://creativecommons.org/licenses/by/4.0/), which permits unrestricted use, distribution, and reproduction in any medium, provided you give appropriate credit to the original author(s) and the source, provide a link to the Creative Commons license, and indicate if changes were made. The Creative Commons Public Domain Dedication waiver (http://creativecommons.org/publicdomain/zero/1.0/) applies to the data made available in this article, unless otherwise stated. 
studies have yet to reach a consensus result on the pain modulatory effects of estrogen. It has been established that OVX rodents developed obvious hyperalgesia and allodynia that could last for as long as 4 months [1], and numerous studies favored an analgesic effect of estrogen on OVX-induced hypersensitivity in intact or inflamed animals [2-8]. However, other literatures mentioned conflicting reports. It has been reported that OVX rats showed a significantly less nociceptive response during the interphase of formalin inflammatory pain, which could be inversely modulated by exogenous estrogen $[5,9,10]$, and estrogen itself was capable of producing hyperalgesia in intact and castrated rats and quails $[11,12]$. Apart from the confusing results derived from behavioral studies, considerable efforts have been devoted to clarify the link between pain modulation by estrogen and the quantitative changes of estrogen receptors. OVX was reported to either downregulate or upregulate estrogen receptors in both brain regions and the spinal cord [13-18]. Concurrently, the auto-regulation of ERs by their ligand has also been extensively studied. While short-term estrogen treatment was reported to differently tune the expression of the receptor subtypes ER $\alpha$ and ER $\beta[19,20]$, chronic estrogen substitution was suggested to decrease the expression of both ERs [14, 21].

The massive contradictions have long been attributed to different timing and dose of estrogen replacement, as well as the tissue of interest and pain modalities examined. However, in retrospect of previous literatures, we noticed that the chronic increase or decrease of estrogen probably weigh far more than the other factors in pain modulation. A pharmacokinetic study by Ito et al. lends further support for this hypothesis [22]. They demonstrated that the universally applied slow-release pellets (Innovate Research, USA) could not maintain a steady systemic estrogen level as was claimed by most studies, in fact the serum estrogen concentration continued to decrease to a significantly lower level compared with that at the time of implantation; thus, it was essentially upon this chronic hormone withdrawal states that most of previous studies based their conclusions. In light of the results, several more complex models were proposed to explore the influence of different estrogen paradigms on neuronal plasticity. Studies indicated that short- or long-term estrogen administration differently determined its analgesic effects [22-24]. Markowska et al. showed that chronic estrogen replacement only when primed with repeated injections was effective in restoration of cognition [25]. However, to our knowledge, none has yet employed a rigorous strategy to investigate the effects of dynamic estrogen alterations on pain regulation. It should be noted that the current studies could not exclude the effects of other hormones and various in the modulation of pain perception, and thus, the results should be interpreted carefully when put into the clinical contexts.

In light of the above observations, we hypothesized that a long-term estrogen change, either with a gradual elevation or decrease in system level, would differently affect pain perception and expression of estrogen receptors. In the present study, we trialed with a bi-directional replacement paradigm to achieve either gradual elevation or decrease of serum estrogen concentration during estrogen replacement in OVX rats, and observed the effects of dynamic estrogen alterations on OVX-induced mechanical hyperalgesia and changes of spinal ER expressions.

\section{Materials and methods \\ Subjects}

Forty adult virgin female Sprague-Dawley rats at the age of 3 months were used. The animals were housed four per cage in a humidity-controlled room with free access to food and water, the temperature of the facility was maintained at $22{ }^{\circ} \mathrm{C}$ with a 12:12-light-dark cycle. The rats were acclimated to manipulation every day during the 7-day-adaptation period to reduce the stress induced by the environment. Rats went through OVX or pellet implantation surgery were housed individually for 7 days and then reformed with their groups. All experimental procedures were carried out in accordance with the National Institute of Health Guide for the Care and Use of Laboratory Animals (NIH Publications No. 86-23). The Animal Ethics and Welfare Committee of Zhejiang University School of Medicine approved all the experimental protocols. An effort was made to minimize the number of animals used and their suffering.

\section{Ovariectomy and estrogen replacement}

Rats were randomly divided into five groups, intact $(n=$ $6)$, sham $(n=6)$, OVX $(n=10)$, E-decrease group $(n=$ $8)$, and E-increase group $(n=5)$. Rats in all groups went through OVX surgery except for the sham group.

After a wash-out period of 20 days after OVX, the rats were implanted with slow-release estrogen pellets or placebo pellets, and the day of OVX surgery was defined as day 0 . All animals underwent surgeries with $1.5 \%$ isoflurane (HeBeiYiPin, China) in oxygen through a nose mask for anesthesia. Briefly, in rats subjected to estrogen replacement, the estrogen pellets or placebo were implanted subscapularly into a skin pocket gently made with the forceps in rats anesthetized with isoflurane, and the pellets were implanted with manufacturer's apparatus (Precision Trochar, Innovate Research of America, US) to minimize the injury. Specifically, the rats in Edecrease group were implanted with one single $2.5-\mathrm{mg}$ estrogen pellet on day 20, and rats in the E-increase group were implanted with one $2.5-\mathrm{mg}$ pellet on day 20 , and then administered with 0.25 -mg pellets on every 
morning of days $33,35,37,39$, and 41 ; at the same time, the rats in E-decrease group were also implanted with the $0.25-\mathrm{mg}$ placebos with the same time frame as in the E-increase group (Fig. 1b). In this way, every $48 \mathrm{~h}$, a $0.25-\mathrm{mg}$ pellet was added, as this interval time was calculated according to the manufacturer's data sheet regarding the drug's half-life and in vivo elimination rate, as well as our verification of the specific metabolite timescale of the pellets (Additional file 4: Figure S3). As a result, the replacement paradigm of the E-decrease and E-increase group was established such that a continuous increase or decrease of serum estrogen trend could be achieved in OVX rats. The rats in the sham group were administered with placebo pellets from the same manufacturer with the same temporal pattern as the E-increase group. All rats were weighted every five days during the experiment.

The slow-release estrogen pellets were purchased from the Innovate Research Company (Innovate Research, US) as the one used in most of previous studies. The temporal and dosing of the replacement of drugs was based on our pilot pharmacokinetic experiments results. Only one kind of drug (slow-release pellet) and delivery method was used in the experiments in order to eliminate any influence of pharmacokinetic variance induced by drug form and delivery routes.

\section{Vaginal cytology}

To monitor the stages of estrus cycle and the effects of estrogen manipulation, vaginal smear of rats were taken daily before the blood extraction and behavioral tests to record the estrous stages at the time of tests. In experiments involving estrogen pellets implantation, vaginal smears were taken before the surgery. A cotton tip dipped with $0.9 \%$ sterile saline was inserted into the vaginal and scrubbed gently, then daubed on a microscope slides. The smears were stained with methane blue and different stages of the estrous cycle were determined using cytology methods according to the description by Freeman (1994). In brief, the pro-estrous was differentiated by a predominance of large, round, nucleated epithelial cells, and an absence of leukocytes, estrus is identified by a majority of cornified squamous epithelial cells, densely distributed leukocytes indicate di-estrous, and met estrus is identified by scattered cells of all three types.

\section{Blood sample and hormone levels quantification}

Blood was sampled every 2 days for animals in all the groups. Under isoflurane anesthesia, 1 1.5-ml blood was collected to a $1.5-\mathrm{ml}$ centrifuge tube from the tail vein of rats, and $1 \mathrm{ml}$ of saline was given to the animal immediately after the blood collection for supplementation of the fluid loss. What's more, during the experiment, all the animals had normal fluid intake and kept gaining weight steadily according to our observations. The blood was allowed to coagulate at room temperature for $2 \mathrm{~h}$ and then centrifuged at $5000 \mathrm{rpm}$ for $10 \mathrm{~min}$ at $-4{ }^{\circ} \mathrm{C}$. After centrifugation, the supernatant was separated into another clean centrifuge tube and frozen at $-20{ }^{\circ} \mathrm{C}$ for

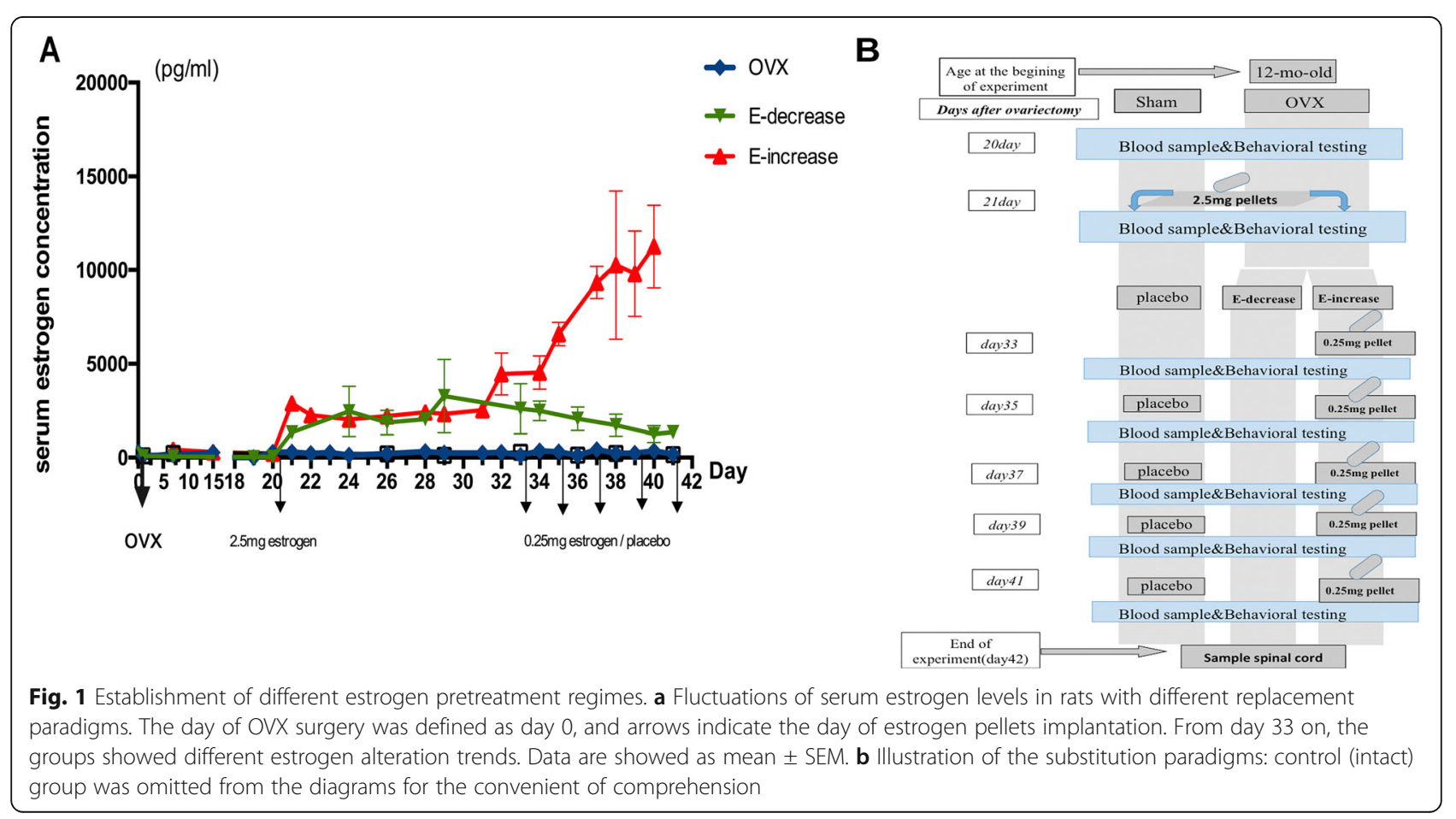


later detection. Estrogen and corticosterone concentration were measured by high-performance liquid chromatography (HPLC). An HPLC system (aglient1290-AB 5500MSD, Agilent Technologies, Calif., USA) was used for chromatographic separation and evaluation, and an equipped software of the HPLC system named Analyst Software was used to record and confirm the spectra peaks in all samples.

\section{Testing of mechanical nociceptive threshold}

Rats in all groups were subjected to electronic von Frey test daily. The von Frey test apparatus (Ugo Basile, Comerio, Italy) consists of three acrylic boxes $(23 \times 18 \times$ $14 \mathrm{~cm}$, each) with a mesh floor through which it was possible to stimulate the plantar with a needle connected to a mechanosensor. The apparatus recorded the paw withdrawal thresholds (PWT) automatically, and it was more accurate and efficient in measuring mechanical sensitivity compared with the traditional von Frey handheld filaments. The behavioral tests were always taken at 10:00 am to reduce any variation related to circadian rhythm. Experiments were conducted in a dark and noise-free room; after a 20-min acclimation, a linearly increasing mechanical force was applied to the rat's dorsal hind paw with the metal von Frey filament. The stimulation force was applied manually in accordance to the synchronized curve provided by the software on the computer. PWL was defined as the force at which the rat withdrew its paw abruptly. Each rat was tested 5 times the most with a 2-min separation, and the three most nearest results with a difference of no more than $10 \mathrm{mN}$ were adopted and averaged. Care was taken not to stimulate the same point of hind paw twice to avoid learning or hypersensitivity and a 20-s cut time was set. A well-trained investigator blind to the group assignment conducted all tests. Spontaneous behaviors (exploratory duration, rearing frequency, and grooming duration) were recorded for 60 min once a week before the von Frey tests.

\section{Immunohistochemistry}

At the last day of the experiments, rats were deeply anesthetized with isoflurane and perfused intracardially with 200 300 ml 4\% formaldehyde in phosphatebuffered saline (PBS). The lumbar (L4 L6) spinal cord was removed and then post-fixed for $4 \mathrm{~h}$ in $4 \%$ paraformaldehyde, cryoprotected overnight in $30 \%$ sucrose in PBS at $4{ }^{\circ} \mathrm{C}$. The spinal cord was then serially sectioned ( $8 \mu \mathrm{m}$ thick) in a transverse plane and mounted on microscope slides. For immunohistochemistry, the sections were stained using avidin-biotin-peroxidase complex technique. Sections were incubated with normal goat serum (Boster, WuHan, China) + 0.3\% Triton-X for $1 \mathrm{~h}$ at room temperature to block non-specific staining.
Slides were then incubated overnight with primary ER antibodies (rabbit anti-ER $\alpha$ polyclonal antibody, 1:100, Abnova, China; rabbit anti-ER $\beta$ polyclonal antibody, 1: 50 , Sigma, USA) in $5 \%$ bovine serum at $4{ }^{\circ} \mathrm{C}$. After washing in PBS, slides were incubated with biotinylated goat anti-rabbit IgG (1:400, Boster, WuHan, China) from the manufacture's kit for $30 \mathrm{~min}$ at room temperature, washed in PBS, and treated with avidin-biotin reagent for $30 \mathrm{~min}$ at room temperature. After three washes in PBS, immunostaining was revealed with $1 \%$ diaminobenzidine tetrahydrochloride, $0.3 \% \quad \mathrm{H}_{2} \mathrm{O}_{2}$ and $1 \%$ nickel chloride in PBS (Boster, WuHan, China). Slides were observed under a microscope and reactions stopped by flushing under tap water when a brown background appeared $(4 \mathrm{~min})$. Slides were then counterstained with hematoxylin (Beyotime, Shanghai, China) for $1 \mathrm{~min}$ and terminated by washes with tap water. Slides were finally dehydrated through graded ethanol solutions followed by xylene and then cover-slipped with neutral balsam (Aladdin, Shanghai, China). The ER $\alpha$ and ER $\beta$ primary antibodies were validated with western blot experiment (Additional file 4: Figure S3b). For immunofluorescence, the GPR30 primary antibody (1:500, rabbit anti-GPR30 polyclonal antibody, Abcam, Hong Kong) was used. Slides were then incubated with FITC-conjugated goat anti-rabbit secondary antibody (1:400, Beyotime, Shanghai, China) for $30 \mathrm{~min}$ at room temperature and stained with 4,6-diamino-2-phenyl indole(DAPI) for nuclei staining. In the control experiments, tissues were processed using the same staining protocol, but omitting the primary antibodies. For quantification of the immunolabeling results, coverslips were viewed using a fluorescence microscope (Eclipse Ni-E, Nikon, Shanghai, China). Images were analyzed using the Image J version 1.50i (National Institute of Health, USA). For quantification of ER positive neurons, slices were taken from each of L4 L6 spinal cord segments of the groups. The optical density of ER positive neurons was analyzed in lamina I and II of the spinal cord. Under a $\times 40$ microscopic field of the slides, a consistent threshold was set on all photographs. After subtracting the background threshold, the software calculated the average optical density of the area of interest. A value of the optical density divided by the number of nuclei in the same area was deemed as the inmmunoactivity of the slides.

\section{Statistics}

All results were presented as mean \pm SEM. The difference of estrogen levels and PWT measures at different time points among groups were analyzed using two-way ANOVA, with Bonferroni's multiple comparisons test for pairwise comparisons. The semi-quantified data of immunohistochemistry of ER expression in each group were compared using 2-tail paired Student $t$ test. 
Pearson's correlation coefficient was used to test for correlations among the different hormonal levels and PWT measures. Statistical analysis was performed using SPSS Statistics Version 22.0 (IBM Corporation, Armonk, NY). $P<0.05$ was considered statistically significant.

\section{Results \\ Establishing a gradual increase or decrease of serum estrogen levels in OVX rats}

Two weeks after ovariectomy, the serum levels of estrogen declined to a very low level, which is close to the minimum detectable by the quantitative assay $(10 \mathrm{pg} /$ $\mathrm{ml}$ ), and this level lasted throughout the experimental range (Fig. 1a). It should be noted that the estrogen levels in both placebo and intact group fluctuated within a normal range $(20 \mathrm{pg} / \mathrm{ml} \sim 150 \mathrm{pg} / \mathrm{ml})$, and were thus not shown in the results. The estrogen treatment paradigms in our experiments differently altered serum estrogen levels. In the E-decrease group, the serum estrogen level was raised in the first 3 days after a single estrogen pellet implantation and remained relatively constant for the next 6 days before a gradual yet significantly decline was observed. At the last day (day 41), the serum concentration in E-decrease group was $1350 \mathrm{pg} / \mathrm{ml}$ on average, $98.3 \%$ of that at the initiation of the substitution (day 20). On the ninth day of the substitution when the estrogen level was the highest in the E-decrease group, the average concentration was $3280 \mathrm{pg} / \mathrm{ml}$ and $238.84 \%$ of that at the initial replacement (day 20).

As for the E-increase group, from the 11th day of the replacement, $0.25-\mathrm{mg}$ pellets were added every $48 \mathrm{~h}$, and this paradigm induced continues acceleration of serum estrogen concentration throughout the later phase of the replacement term. The highest estrogen concentration of the E-increase group was reached at the end of the experiment at $11255 \mathrm{pg} / \mathrm{ml}$ and almost eight times higher than that of the E-decrease group. According to pharmacokinetic observations in our pilot study, a single 0.25 - mg pellet implanted in OVX rats would result in a rapid serum estrogen surge that peaked at $24 \mathrm{~h}$ after implantation and lasted for at least 2 days, and then it decreased rapidly to approximately physiological serum level at day 21 (Additional file 2: Figure S1). Thus, $48 \mathrm{~h}$ was supposed to be the approximate half-life of the 0.25 mg drug pellet under the current replacement condition. Our results demonstrated that with a carefully designed replacement strategy, both chronic increase and decease of estrogen levels in OVX rats within a 21-day frame could be achieved. This model could serve as a tool for our investigation of the effects of bi-directional hormone changes on pain modulation.

Considering the potential effects of multiple blood collection on the HPA axis and animal's stress levels, we tested the serum corticosterone concentration as well as evaluated the grooming durations of animals in different groups. Our results showed that the blood extraction procedure did not have significant effects on rat's corticosterone levels when compared with their initial levels during the experiment (Additional file 3: Figure S2). In addition, the behavioral results showed that the grooming duration was higher in OVX compared with the control group, which was reduced by estrogen replacement (Additional file 1: Table S1). Taken together, these results further confirmed that the multiple blood collection method did not have significant stressful influence on the animals in the experimental regime.

\section{Estrogen decline or elevation during estrogen replacement differently modulates mechanical hypersensitivity in OVX rats}

ANOVA analysis revealed evidently different trends in the alterations of the PWT among different estrogen replacement groups. We did not observed any significant changes of the PWT in female rats at different phases of estrous cycle in a previous study (data not shown), the PWT falls within $33 \sim 38 \mathrm{mN}$ as were observed in our experiments. Our results demonstrated that OVX rats developed a significant mechanical hyperalgesia (Fig. 2a), the PWT progressively decreased starting from 1 week after the OVX surgery to as low as $40.3 \%$ of the control level, and this hyperalgesia state in rats lasted until the end of our experiment. In the E-decrease group where rats were replaced with a single $2.5-\mathrm{mg}$ pellet on day 20 , the OVX-induced hyperalgesia was reversed immediately 2 days after estrogen implantation. Their PWT measures recovered gradually to a point approximate to the level before the ovariectomy at the end of the replacement session (day 41). This result matches with those of previous studies proving estrogen's antihyperalgesia effects. Two-way ANOVA revealed that during the early phase (from day 20 to day 33) of estrogen replacement, the pain threshold in the Edecrease group increased rapidly, and was then maintained at a relatively steady state, while during the later phase of the replacement (from day 33 to day 41), the PWT increased even more dramatically compared with that of the early phase (Fig. 2b).

Concurrently, the E-increase group also reversed the OVX-induced mechanical hyperalgesia during the early phase of the $2.5-\mathrm{mg}$ pellet replacement as was with the E-decrease group. Surprisingly, starting from day 34 when the first $0.25-\mathrm{mg}$ pellet is implanted, the PWT in the E-increase group took a decreasing trend and this downregulation continued with the other 40.25 -mg pellets implanted in succession. At the end of the experiment (day 41), the PWT in the E-increase group was significantly lower than that in the E-decrease group 


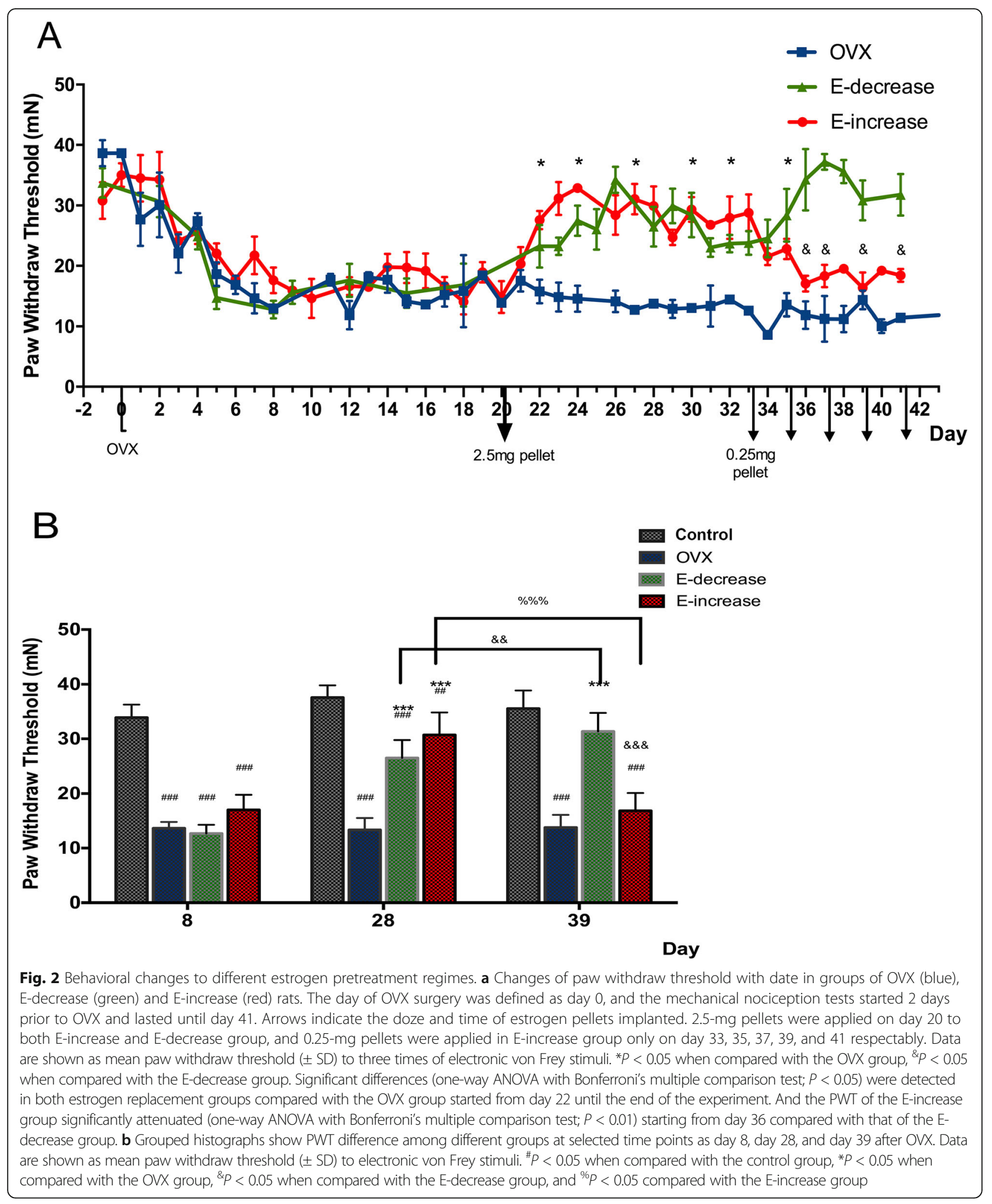

(57.9\%), and maintained at a level close to that in the OVX rats (Fig. 2b). In addition, during the entire range of the experiment, the E-increase group did not show pain threshold significantly higher than that of the E-decrease group at any time point, despite the fact that their serum estrogen concentration has been significantly higher than the E-decrease group. Collectively, these results demonstrated that (1) estrogen replacement reversed the OVX- 
induced hyperalgesia in a dose-independent manner and (2) the late phase estrogen decline or elevation during estrogen replacement differently modulated mechanical hypersensitivity, as the late phase estrogen decrease fully reversed the OVX-induced mechanical hyperalgesia, while the late phase estrogen increase inflicted an contrary effect and abolished the early phase estrogenic analgesia. Furthermore, to investigate if there is a correlation between the serum estrogen concentrations and the PWT values, we conducted Pearson's correlation analysis. The resulting Pearson's correlation coefficient revealed a negative correlation between the PWT and estradiol serum levels in the OVX group ( $n=24, r=-0.53, P=0.0083)$. However, there were no significant correlations between the PWT and estrogen levels in the E-decrease and the E-increase groups during the estrogen replacement phase (day 20 day 41). The results suggested that estrogen levels at a given time point was not correlated with the mechanical nociception under the current estrogen substitution circumstances (Fig. 3). In addition, a single 0.25-mg estrogen replacement also attenuated OVX-induced hyperalgesia with a rapid reduced serum estrogen levels starting from pellet implantation (Additional 4: Figure S3).

Estrogen decline or elevation during estrogen replacement differently modulates estrogen receptor expression

At the end of measuring the PWT, the expression of estrogen receptors in the spinal cord was evaluated and compared among different groups. It should be noted that at the lumbar spinal cord of rats, ERs were mainly distributed in lamina I II, which was verified in our results (Fig. 4). ER $\alpha$ showed a more predominant expression than ER $\beta$ in control sham-operated rats. In comparison with the control group, long-term OVX significantly reduced the expression of ER $\alpha$ (Fig. 4a, c, $P<0.05$ ), while ER $\beta$ was not affected significantly (Fig. 4b, d). These results were in agreement with a previous study [16], which reported ovariectomy decreased ER $\alpha$ but not ER $\beta$ mRNA levels in rat spinal cord. Post hoc comparison revealed that estrogen replacement in E-decrease group significantly increased ER $\alpha$ expression compared with the OVX group $(P<0.001)$, while the expression of ER $\beta$ was hardly influenced (Fig. 4c, d). Interestingly, in E-increase group, the results showed that the late phase estrogen surge dramatically upregulated the expression of ER $\alpha$ compared with all the other groups $(P<0.001$ vs. sham, OVX, Edecrease group); simultaneously, the expression of ER $\beta$ was strongly depressed in the spinal cord at the last day of the replacement. To view the change more clearly, the expression ratio of the two receptors was compared among different groups, and the results showed that rats in the Eincrease group manifested the largest $E R \alpha / E R \beta$ ratio (Fig. 4e). Furthermore, we also evaluated the expression of membrane estrogen receptor GPR30, and the results showed that different estrogen fluctuation modes did not inflict significant effects on GPR30 protein expression (Fig. 5). Negative controls omitting the first antibodies resulted in no positive stains (Additional file 5: Figure S4), and all the observations of ERs positive stains were conducted on the L4 L6 spinal cord segments (Additional file 6: Figure S5).

\section{The effects of estrogen replacement on vaginal smear and weight change}

OVX induced a di-estrus characteristic of the rat vaginal smear (Table 1), while estrogen replacement induced a

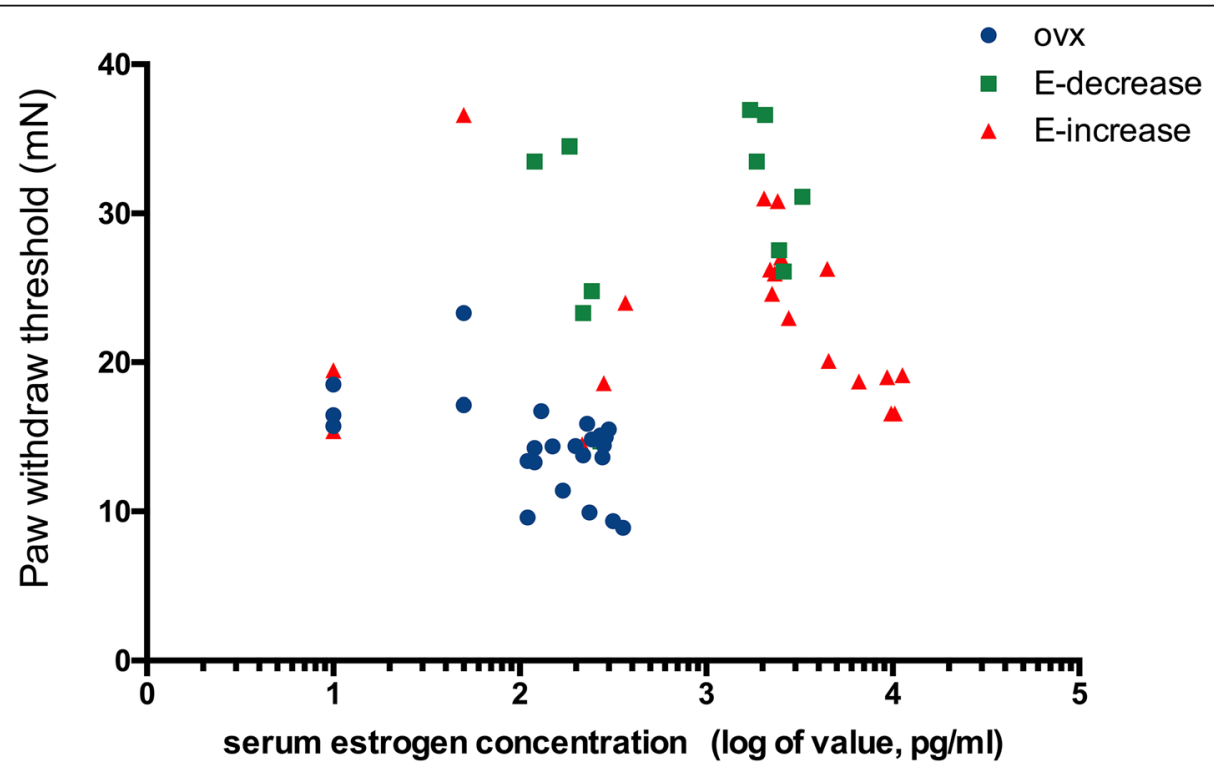

Fig. 3 Correlation between serum estrogen and PWT. Analysis of correlation between serum estrogen levels and the PWT value of each group (Pearson's correlation coefficient). Data are shown as mean \pm SD 
A
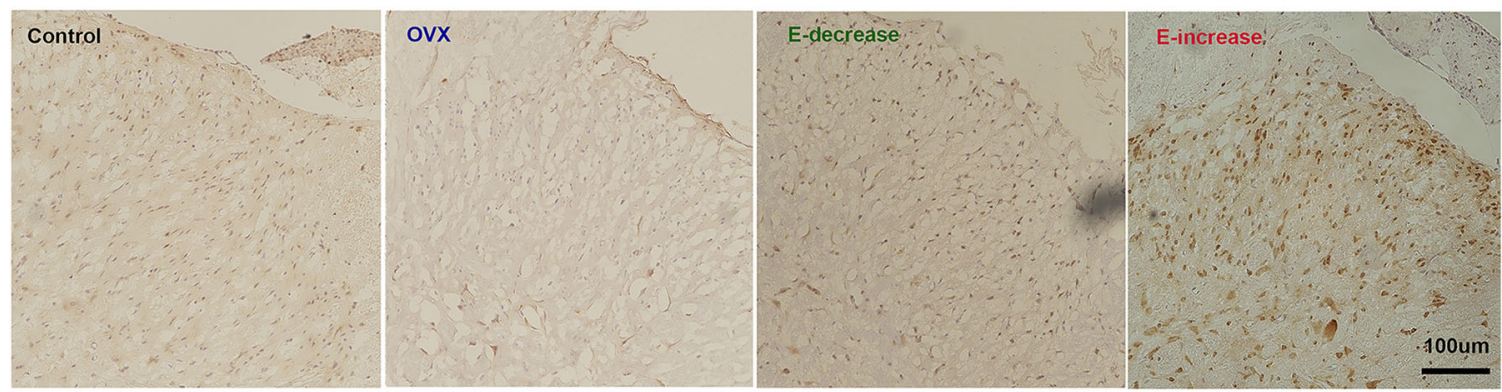

B
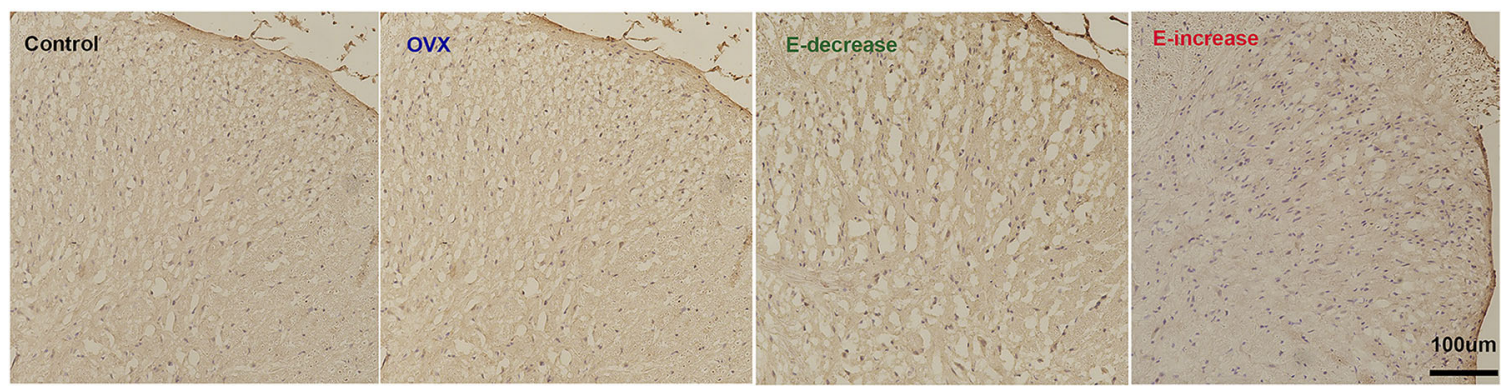

C

D
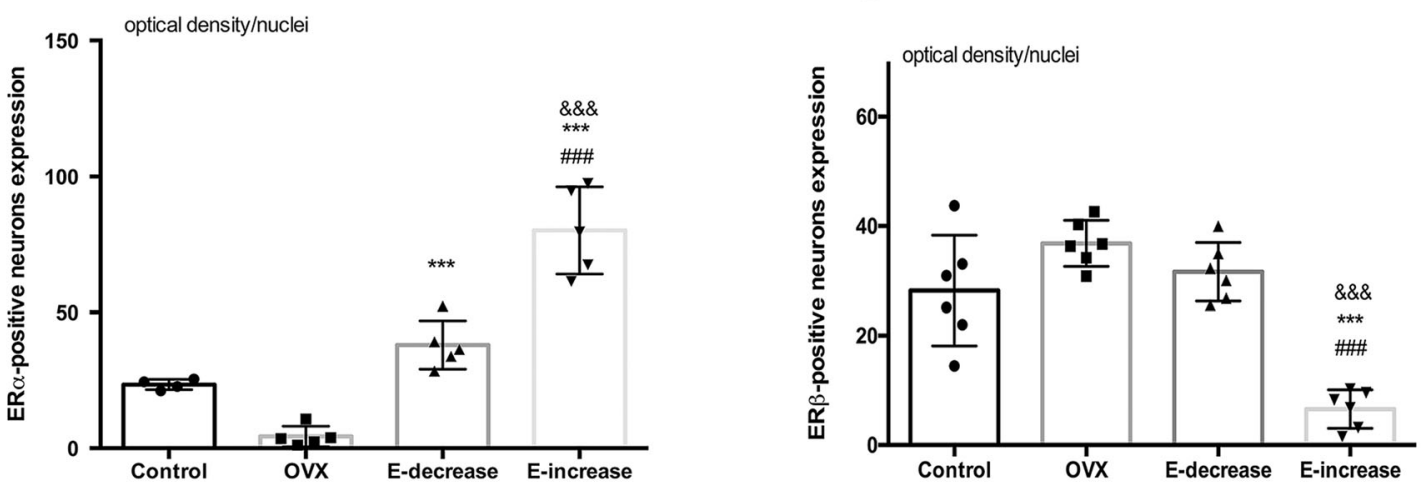

E

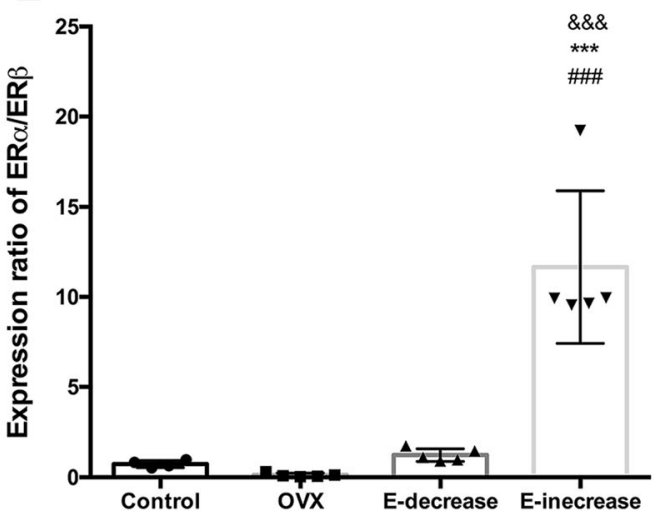

Fig. 4 (See legend on next page.) 
(See figure on previous page.)

Fig. 4 Expression change of ERa and ER $\beta$ to different estrogen pretreatment regimes. Expression of $\mathbf{a} E R a$ and $\mathbf{b}$ ER $\beta$ positive neurons in rat spinal cord at the last day of estrogen substitution. ER positive neurons mainly concentrated in the laminae I $\sim \|$ of the spinal cord, pictures are shown at $\times 20$ magnification, scale bar represents $100 \mu \mathrm{m}$. c, d Histographs show one-way ANOVA analysis of the difference of ER expression among groups. Data are shown as mean \pm SD. ${ }^{*} P<0.05$ when compared with the OVX group, ${ }^{*} P<0.05$ when compared with the E-decrease group. e Expression ratio of ERa to ERß of each group

prevalence of cornified cells in the rat vaginal smear which represents estrus, as reported by a previous study [25]. At the beginning of the experiment, all rats were 3 months old and weighed at 240 260 g. Three weeks after OVX, rats in all groups accumulated weight (Additional file 7: Figure S6). The rats in OVX group significantly gained weight throughout the course of experiment, and at the end of the experiment, their average weight was significantly higher than that of rats in the control group. The gain in body weight in OVX rats was reversed easily by both modes of estrogen treatment, and at the end of experiment, there was no difference between the Edecrease/increase and the control groups.

\section{Discussion}

The present study demonstrated that chronic and continuous estrogen increase or decrease in OVX rats differently affect OVX-induced hypersensitivity in these animals. With our replacement strategies, continuous decline of estrogen at a late stage reversed mechanical hyperalgesia, while progressive elevation of estrogen abrogated its analgesic effect and progressively attenuated mechanical threshold compared with the control group. Coincided with the behavioral changes, chronic estrogen downregulation results in increased ER $\alpha$ and ER $\beta$ expression in the spinal cord, while chronic upregulation of estrogen increased spinal ER $\alpha$ more significantly and dramatically reduced ER $\beta$.
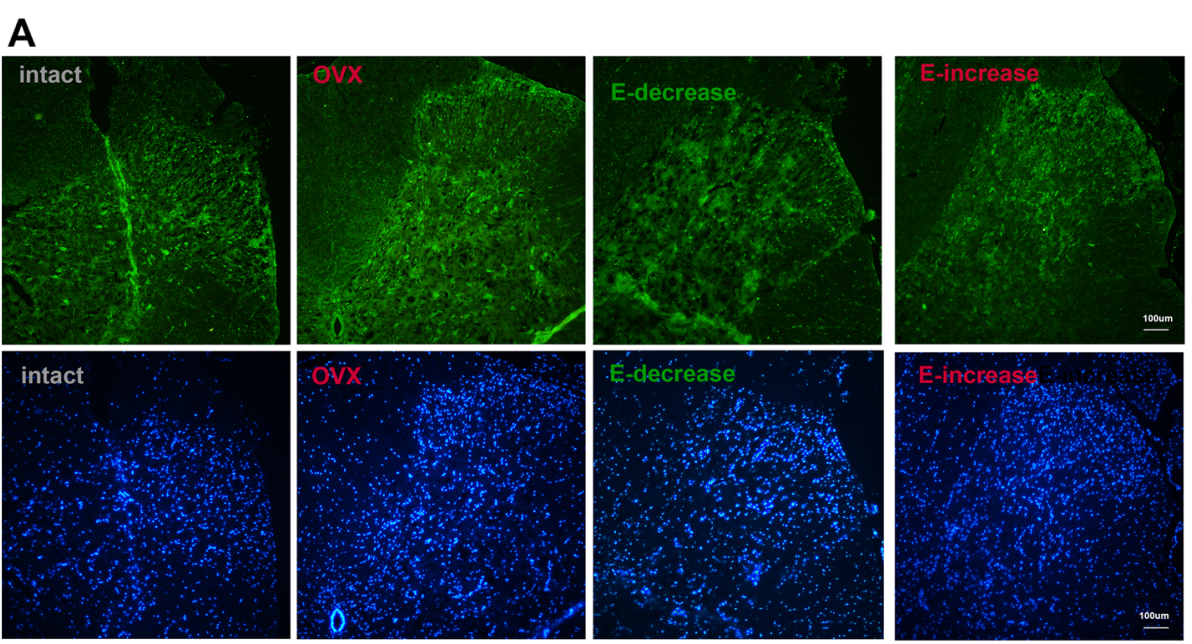

B

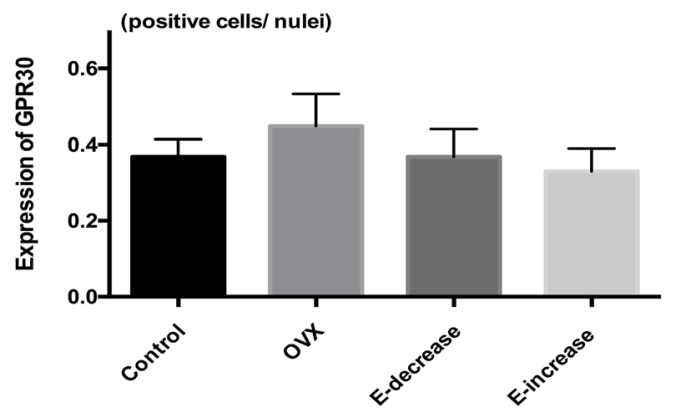

Fig. 5 Change of expression of GPR30 under different estrogen pretreatment regimes. a GPR30 positive neurons in the spinal cord in rats of different groups at the end of the experiment. Pictures were shown at x 10 magnification. Scale bar represents $100 \mu \mathrm{m}$. b Histographs show oneway ANOVA analysis of the difference of GPR30 expression among groups. Data are shown as mean \pm SD, and no significance was detected among the groups 
Table 1 Classification of the estrous cycle of rats in different groups

\begin{tabular}{llllll}
\hline & Day3 & Day21 & Day26 & Day37 & Day41 \\
\hline Control & pro/es/de & pro/es/de & pro/es/de & pro/es/de & pro/es/de \\
OVX & de & de & de & de & de \\
E-decrease & de & pre/es & es & es & pre/es \\
E-increase & de & pre/es & es & es & es \\
\hline
\end{tabular}

pro, proestrus; de, di-estrus; pre, pre-estrus; es, estrus

It is proposed that the discrepancy regarding estrogen modulation on pain-related behaviors was due to various pain modalities examined and different doses and delivery methods of estrogen used. Based on our and previous results, the inconsistency may be partly explained by chronic estrogen increase or decrease in the animals. Studies investigating rat recognition and memories showed that cyclic rather than continuous estrogen replacement facilitated the acquisition of spatial memory [26]. And chronic estrogen replacement regimes differently affect restoration of cognition [25]. However, the aforementioned studies adopted more than one kind of drug formulations or delivery methods (i.p. injection and pellet implantation), which would inevitably influence the consistency of drug pharmacokinetics among different experimental groups, and thus add another variable to the already complicated mechanisms through which estrogen exert its neuronal modulation effects. In addition, several early studies claimed to obtain sustained serum levels of estrogen with implantation of silastic capsules, yet this method was not adopted widely due to the inefficient drug release from the silastic tube [27]. In the present study, we chose the widely used slow-release estrogen pellets and managed to achieve continuous estrogen increase or decrease trends through careful experimental design; therefore, the approach described here may be superior to those using other drug forms. Furthermore, our pilot study results verified that the widely used estrogen pellets, do provide a consistent release rate in vivo; however, the actual estrogen serum concentration fluctuated dramatically and failed to maintain a steady level, this could be explained by the dynamic metabolism of serum hormones in vivo as well as an accumulation effect in distal tissues.

In summary, the present study is the first to explore the influence of different trends of gradual estrogen change on pain modulation. The most important finding of the present study is that gradual decrease rather than increase of serum estrogen levels was capable of reversing OVX-induced hyperalgesia. Moreover, the mechanical threshold even decreased in the E-increase group to a significantly lower level compared with that of the Edecrease group during the later phase of the experiment, which indicated that continues increasing trend of estrogen level abolished its analgesic effects, and tend to re-establish the mechanical hyperalgesia induced by OVX. These results should enlighten us in that chronic estrogen fluctuation consists of a far more predominant component in pain modulation than was expected, especially under a hypersensitive state in female rats. These results appears to have implications for clinical findings in patients with chronic pain disorders, dynamic decrease in blood estrogen levels have been noted in cases of multiple pain disorders including perimenstrual migraine, and estrogen replacement therapy was associated with risk of orafacial and back pain in women [22]. It remained to be elucidated whether a causal relationship could be established between estrogen increase or decrease trends and modulation of clinical pain-related syndromes in women. Plenty of studies have examined the roles of estrous cycle in somatic and visceral pain modulation $[6,28,29]$. Robbins et al. showed that a sudden estrogen withdrawal achieved by brief estrogen pellet implantation produced a robust increase in VMRs evoked by bladder distension [24], and proestrus-like surge of serum estrogen increased inflammation-evoked nociception in female rats [30]. However, it is intrinsically difficult to generalize the findings based on rat estrus cycle to human. And the short-term alteration of hormone levels was beyond the scope of the present study.

Our results demonstrated that OVX generated persistent mechanical hyperalgesia within 1 week, and a single estrogen pellet replacement was capable of reversing the OVX-induced hypersensitivity. These results were in agreement with previous studies $[2,4,5]$, which showed that OVX induced a hyperalgesic state that was deemed as a kind of chronic pain. Thermal nociception was seldom affected as was concluded by Sanoja et al [2]; however, based on our observations, OVX rats showed a trend of decreased thermal pain threshold 3 weeks following the surgery (data not shown). It should be reiterated that the estrogen dose used in our study $(2.5 \mathrm{mg}$, 21-day release pellet) was slightly higher than that in most previous studies; nevertheless, the mechanical hyperalgesia was alleviated dramatically following replacement, which indicates that the estrogenic analgesia effects was dose-independent. In addition, considering that stress levels could also affect animal pain perception, and the multiple blood collection process in our experiments may influence the HPA axis in rats, we also tested the serum corticosterone levels and the grooming duration of rats among different groups during the experiment. Our results are consistent with previous studies in that OVX rats have higher levels of serum corticosterone compared with intact rats [7], and estrogen replacement reduced their stress levels, which may offer some explanation for the nociception difference 
under different hormone states. However, most importantly, the blood collection process in our experiment has no significant effect on rat corticosterone levels when assessed during the 2-week experiment.

The immunohistochemistry results showed that longterm OVX decreased spinal ER $\alpha$ expression while ER $\beta$ was less affected. Previous literatures implicated that the change of ERs may be time-dependent after OVX in rats. For short-term OVX upregulated ERs genes accurately in female rats [14], and long-term OVX gradually downregulated ERs in rat brain [13, 15]. Our observations are in consistent with the studies concerning long-term OVX. Most importantly, our results showed that spinal ERs were differently regulated under the two estrogen replacement regimes as was assessed at the end of the experiment. In the E-decrease group, ER $\alpha$ increased significantly after the 21-day hormone substitution while ER $\beta$ was slightly decreased but without significance compared with the control group. These results conflict a previous study, which recorded decrease of both ERs after estrogen replacement [14] . The confliction seems more likely to be a result of different timing of the evaluation of ERs change rather than of different estrogen concentration. Due to the current method limitation, we were unable to monitor the ERs change dynamically throughout the replacement course. On the other hand, in the E-increase group, the expression of spinal ER $\alpha$ was increased even more dramatically, while ER $\beta$ was strongly depressed compared with the E-decrease and the control groups. These results indicate that the disparate findings at behavioral level may be linked to the distinctive modulation of central level ERs.

Despite the incoherent results, ER $\alpha$ is mostly associated with classic reproductive-related functions and its role related to pain modulation is conflicting, while ER $\beta$ was more predominantly involved in inhibitory effects on nociception in various pain modalities [31,32], and ablation of either $E R \alpha$ or ER $\beta$ would eliminate the sex difference in mechanical nociception in normal and inflamed mice [33]. At the central level, ER $\alpha$ is found to co-localized with preproenkephalin mRNA and estrogen could rapidly increase spinal enkephalin levels $[8,34]$. ER $\alpha$ activation was concluded to be antinociceptive in the formalin model [35]; at the same time, it exaggerated visceral pain in several conditions [36]. Khomula et al. proved that ER $\alpha$ agonist facilitates hyperalgesic priming though IP3 receptor by electrophysiological means [37], and ER $\alpha$ antisense attenuated AMP-induced hyperalgesia in primed female rats [38]. These studies collectively confirmed that ER $\alpha$ is involved in central pain pathways. It is possible that the over-activation of ER $\alpha$ further facilitated the hyperalgesia in OVX rats, as was supposed in the E-increase group. Anatomical studies showed ER $\beta$ was co-localized with GABAergic neurons through which it may adjust the inhibitory tune at the spinal level $[32,39]$. ER $\beta$ is also reported to enhance the serotonergic
(5-HT) descending inhibitory pathway in rat dorsal raphe nuclei [40]. Together, these results provide fundamental basis for ER $\beta$ to exert its analgesic effects during pain transduction. Piu et al. found that ER $\beta$ agonists protect from inflammatory pain as well as $\mathrm{PEG}_{2^{-}}$or capsaicininduced hyperalgesia in rats [31, 41-43], and ER $\beta$ knockout mice manifested a hyperalgesic phenotype in resemblance of human interstitial cystitis syndromes [44]. Though contradictions exist $[35,45]$, overall ER $\beta$ is capable of alleviating both acute and chronic pain in a relatively ligand-specific manner [46]. We may postulate that the hyperalgesia showed in the E-increase group could be correlated to a depletion of ER $\beta$ at the central sites which eliminated its analgesic effects in OVX states.

Despite the multiple signaling pathways implicated in ERs modulation of pain, it is suggested that estrogen's regulation effects could be deemed as a balance between the two opposing forces derived from the two-receptor subtypes. Induced expression of ER $\beta$ is revealed to have bi-directional effects on ER $\alpha$ regulated genes, enhancing or counteracting the effects of ER $\alpha$ in vitro and in vivo [47-49], ER $\alpha: E R \beta$ ratio differently affected OVXinduced osteoporotic fracture healing [50]. It is reasonable that the conflicting behavioral results may be attributed to the different expression ratio of central $E R \alpha: E R \beta$ accompanying fluctuating levels of estrogen. As has been showed in our results, the disparity in pain responsiveness induced by estrogen was pertinent to different spinal ER $\alpha: E R \beta$ expression ratio. It could be anticipated that a mismatch modulatory action of estrogen receptors may lead to a hyperalgesic state as was seen in the E-increase group in the present study.

Estrogen could activate both classical ERs and membrane ERs following activation of its receptors, and both pathways could result in long-term changes in neuronal plasticity and nociception. It has been increasingly recognized that membrane ERs may be more extensively engaged in pain transduction [51]. ERs in brain regions are organized with mGluRs to activate protein kinase A, protein kinase $\mathrm{C}$, and mitogen activated protein kinase (MAPK) cascades [52-54]. However, based on our present observations, it is hard to determine whether estrogen exert its different modulatory effects through which ER-related pathways, and further studies is warranted to elucidate the mechanisms involved.

\section{Perspectives and significance}

The present study investigated the effects of continuous increase or decrease of in vivo estrogen level in a hyperalgesia model of OVX. Our results revealed that estrogen's nociceptive effect is not only dependent on its presents or not but also on its dynamic change of system level, and this may in some extent explain the contradictions concerning estrogen's anti-nociception or pro-nociception effects as demonstrated by previous studies; thus, researchers should 
take into consideration of the effects of estrogen's dynamic change of level in the future studies.

\section{Conclusion}

The present study extends previous findings concerning the effects of estrogen on nociception. Our results highlight the pivotal role of chronic estrogen increase or decrease in pain modulation from a dynamic point of view. The different mechanism activated by the hormone level change remains to be elucidated.

\section{Supplementary information}

Supplementary information accompanies this paper at https://doi.org/10. 1186/s13293-019-0271-5.

Additional file 1: Table S1. Spontaneous behavioral results of rats in different hormone states.

Additional file 2: Figure S1. Pharmacokinetic experiments with estrogen pellets. Alterations of serum estrogen concentration when implanted with one single $0.25 \mathrm{mg}$ pellet in rats.

Additional file 3: Figure S2. Effects of blood collection on serum corticosterone levels. Change of serum corticosterone levels in rats with different replacement paradigms. The day of OVX surgery was defined as day 0 , and arrows indicate the day of estrogen pellets implantation. Corticosterone levels didn't show significant changes in each group when compared with their own initial concentrations respectively. Data are showed as mean \pm SEM.

Additional file 4: Figure S3. Effects of physiological level estrogen pellets implantation. (A) Serum estrogen levels when implanted with one single 0.25 -mg pellet. (B) Change of PWT in rats of different estrogen treatment groups. Data are shown as mean paw withdraw threshold $( \pm$ SD) to three times of electronic von Frey stimuli.

Additional file 5: Figure S4. Negative control. (A)

Immunohistochemistry results of negative control and positive control. (B) Validation of primary antibodies with western blot. ERa and ERß were detected at molecular weight of $66 \mathrm{kd}$ and $55 \mathrm{kd}$ respectively.

Additional file 6: Figure S5. Low-magnification images show the $L 4 \sim L 6$ spinal segments in which all the observations of ERs were conducted. And high-magnification images for differentiate the between the counter stain and the DAB stain. (A) Stain of ERa (B) Stain of ER $\beta$ of the $L 4 \sim L 6$ spinal segments.

Additional file 7: Figure S6. Change of body weight in different groups. Time course of body weight of the rats in one of the following assigned groups: control (black), ovariectomized (blue), E-increase (red), E-decrease (green). Age at the start of the curve was 12 weeks.

\section{Acknowledgments}

We would like to thank Professor Shucai Ling (Zhejiang University School of Medicine, Zhejiang, China) for his technological guidance in the experiments performed as part of this study.

\section{Authors' contributions}

WZ participated in the design of the study, conducted the behavioral experiments and drafted the manuscript. HW and QX participated in the behavioral experiments. SC, LS, and CJ carried out the immunohistochemistry studies. LW, FF, XQ, and YF participated in the data collection and performed the statistics, XC conceived of the study, and participated in its designing and coordination and helped to draft the manuscript. All authors read and approved the final manuscript.

\section{Funding}

This work was supported by the National Natural Science Foundation of China (NSFC, No. 81271237, No. 81471126, and No. 81501702).

\section{Availability of data and materials}

Data sharing not applicable to this article as no data-sets were generated or analyzed during the current study.

\section{Ethics approval}

All experimental procedures were carried out in accordance with the National Institute of Health Guide for the Care and Use of Laboratory Animals (NIH Publications No. 86-23). The Animal Ethnics and Welfare Committee of Zhejiang University School of Medicine approved all the experimental protocols.

\section{Consent for publication}

Not applicable.

\section{Competing interests}

The authors declare that they have no competing interests.

\section{Author details}

${ }^{1}$ Department of Anesthesia, Women's Hospital, Zhejiang University School of Medicine, Xueshi Road 1, Hangzhou 310006, China. ${ }^{2}$ Zhejiang University School of Medicine, 866th Yuhangtang Road, Hangzhou 310058, China.

Received: 23 May 2019 Accepted: 21 November 2019

Published online: 09 January 2020

References

1. Pajot J, Ressot C, Ngom I, Woda A. Gonadectomy induces site-specific differences in nociception in rats. Pain. 2003;104(1-2):367-73.

2. Sanoja R, Cervero F. Estrogen-dependent abdominal hyperalgesia induced by ovariectomy in adult mice: a model of functional abdominal pain. Pain. 2005;118(1-2):243-53. https://doi.org/10.1016/j.pain.2005.08.021.

3. Estrogen modulation of ovariectomy-induced hyperalgesia in adult mice: Eur J Pain, 2007. (Jul, 2008).

4. Ceccarelli I, Fiorenzani P, Massafra C, Aloisi AM. Long-term ovariectomy changes formalin-induced licking in female rats: the role of estrogens. Reprod Biol Endocrinol. 2003;1:24.

5. Kuba T, Kemen LM, Quinones-Jenab V. Estradiol administration mediates the inflammatory response to formalin in female rats. Brain Res. 2005;1047(1): 119-22. https://doi.org/10.1016/j.brainres.2005.04.005.

6. Kramer PR, Bellinger LL. The effects of cycling levels of 17 beta-estradiol and progesterone on the magnitude of temporomandibular joint-induced nociception. Endocrinology. 2009;150(8):3680-9. https://doi.org/10.1210/en. 2008-1707.

7. Mannino CA, South SM, Quinones-Jenab V, Inturrisi CE. Estradio replacement in ovariectomized rats is antihyperalgesic in the formalin test. J Pain. 2007:8(4):334-42. https://doi.org/10.1016/j.jpain.2006.10.002.

8. Sanoja R, Cervero F. Estrogen modulation of ovariectomy-induced hyperalgesia in adult mice. Eur J Pain. 2008;12(5):573-81. https://doi.org/10. 1016/j.ejpain.2007.09.003

9. Gaumond I, Arsenault P, Marchand S. Specificity of female and male sex hormones on excitatory and inhibitory phases of formalin-induced nociceptive responses. Brain Res. 2005;1052(1):105-11. https://doi.org/10. 1016/j.brainres.2005.06.011.

10. Gaumond I, Arsenault P, Marchand S. The role of sex hormones on formalin-induced nociceptive responses. Brain Res. 2002;958(1):139-45.

11. Zhao $L$, Brinton RD. Estrogen receptor alpha and beta differentially regulate intracellular $\mathrm{Ca}(2+)$ dynamics leading to ERK phosphorylation and estrogen neuroprotection in hippocampal neurons. Brain Res. 2007;1172:48-59. https://doi.org/10.1016/j.brainres.2007.06.092.

12. Evrard HC, Balthazart J. Aromatization of androgens into estrogens reduces response latency to a noxious thermal stimulus in male quail. Horm Behav. 2004;45(3):181-9. https://doi.org/10.1016/j.yhbeh.2003.09.014.

13. Ito H, Uno T, Kawata T, Isobe K. Radiation therapy effects in ovarian cancer-review. Nihon Rinsho. 2004;62(Suppl 10):560-4.

14. Mohamed MK, Abdel-Rahman AA. Effect of long-term ovariectomy and estrogen replacement on the expression of estrogen receptor gene in female rats. Eur J Endocrinol. 2000;142(3):307-14

15. Papka RE, Storey-Workley M, Shughrue PJ, Merchenthaler I, Collins JJ, Usip S, et al. Estrogen receptor-alpha and beta- immunoreactivity and mRNA in neurons of sensory and autonomic ganglia and spinal cord. Cell Tissue Res. 2001;304(2):193-214. 
16. Nishikawa $Y$, Ikegami H, Sakata M, Mizutani T, Morishige K, Kurachi H, et al. Ovariectomy increases the level of estrogen receptor mRNA and estrogen receptor binding sites in female rat adipose tissue. J Endocrinol Invest. 1993; 16(8):579-83. https://doi.org/10.1007/bf03347674.

17. Rose'Meyer RB, Mellick AS, Garnham BG, Harrison GJ, Massa HM, Griffiths LR. The measurement of adenosine and estrogen receptor expression in rat brains following ovariectomy using quantitative PCR analysis. Brain Res Brain Res Protoc. 2003;11(1):9-18.

18. Lim SK, Won YJ, Lee HC, Huh KB, Park YS. A PCR analysis of ERalpha and ERbeta mRNA abundance in rats and the effect of ovariectomy. J Bone Miner Res. 1999;14(7):1189-96. https://doi.org/10.1359/jbmr.1999.14.7.1189.

19. Taleghany N, Sarajari S, DonCarlos LL, Gollapudi L, Oblinger MM. Differential expression of estrogen receptor alpha and beta in rat dorsal root ganglion neurons. J Neurosci Res. 1999;57(5):603-15.

20. Sohrabji F, Miranda RC, Toran-Allerand CD. Estrogen differentially regulates estrogen and nerve growth factor receptor mRNAs in adult sensory neurons. J Neurosci. 1994;14(2):459-71.

21. Chen C, Kalu DN. Modulation of intestinal estrogen receptor by ovariectomy, estrogen and growth hormone. J Pharmacol Exp Ther. 1998;286(1):328-33.

22. Ito A, Takeda M, Furue H, Shibata K, Hori M, Sagai H, et al. Administration of estrogen shortly after ovariectomy mimics the anti-nociceptive action and change in 5-HT1A-like receptor expression induced by calcitonin in ovariectomized rats. Bone. 2004;35(3):697-703. https://doi.org/10.1016/j.bone.2004.04.012.

23. Niyomchai T, Jenab S, Festa ED, Akhavan A, Quinones-Jenab V. Effects of shortand long-term estrogen and progesterone replacement on behavioral responses and c-fos mRNA levels in female rats after acute cocaine administration. Brain Res. 2006;1126(1):193-9. https://doi.org/10.1016/j.brainres.2006.07.099.

24. Robbins MT, Mebane $H$, Ball CL, Shaffer AD, Ness TJ. Effect of estrogen on bladder nociception in rats. J Urol. 2010;183(3):1201-5. https://doi.org/10. 1016/j.juro.2009.11.003.

25. Markowska AL, Savonenko AV. Effectiveness of estrogen replacement in restoration of cognitive function after long-term estrogen withdrawal in aging rats. J Neurosci. 2002;22(24):10985-95.

26. Lipatova O, Byrd D, Green JT, Toufexis DJ. Effects of continuous vs. cycling estrogen replacement on the acquisition, retention and expression of placeand response-learning in the open-field tower maze. Neurobiol Learn Mem. 2014;114:81-9. https://doi.org/10.1016/j.nlm.2014.05.001.

27. Mannino CA, South SM, Inturrisi CE, Quinones-Jenab V. Pharmacokinetics and effects of 17beta-estradiol and progesterone implants in ovariectomized rats. J Pain. 2005;6(12):809-16. https://doi.org/10.1016/j.jpain.2005.07.007.

28. Lu YC, Chen CW, Wang SY, Wu FS. 17Beta-estradiol mediates the sex difference in capsaicin-induced nociception in rats. J Pharmacol Exp Ther. 2009;331:3:1104-1110; doi:https://doi.org/10.1124/jpet.109.158402.

29. Zhao Q, Tan Z, Guo J, Chen Y. Estrous cycle-dependent expression of estrogen receptor alpha in periodontal tissue. Chronobiol Int. 2007;24(3): 425-33. https://doi.org/10.1080/07420520701420410.

30. Ralya A, McCarson KE. Acute estrogen surge enhances inflammatory nociception without altering spinal Fos expression. Neurosci Lett. 2014;575: 91-5. https://doi.org/10.1016/j.neulet.2014.05.035.

31. Piu F, Cheevers C, Hyldtoft L, Gardell LR, Del Tredici AL, Andersen CB, et al. Broad modulation of neuropathic pain states by a selective estrogen receptor beta agonist. Eur J Pharmacol. 2008;590(1-3):423-9. https://doi.org/ 10.1016/j.ejphar.2008.05.015.

32. Blurton-Jones M, Tuszynski MH. Estradiol-induced modulation of estrogen receptor-beta and GABA within the adult neocortex: a potential transsynaptic mechanism for estrogen modulation of BDNF. J Comp Neurol. 2006;499(4):603-12. https://doi.org/10.1002/cne.21122.

33. Li L, Fan X, Warner M, Xu XJ, Gustafsson JA, Wiesenfeld-Hallin Z. Ablation of estrogen receptor alpha or beta eliminates sex differences in mechanical pain threshold in normal and inflamed mice. Pain. 2009;143(1-2):37-40. https://doi.org/10.1016/j.pain.2009.01.005.

34. Amandusson A, Hermanson O, Blomqvist A. Colocalization of oestrogen receptor immunoreactivity and preproenkephalin mRNA expression to neurons in the superficial laminae of the spinal and medullary dorsal horn of rats. Eur J Neurosci. 1996;8(11):2440-5.

35. Coulombe MA, Spooner MF, Gaumond I, Carrier JC, Marchand S. Estrogen receptors beta and alpha have specific pro- and anti-nociceptive actions. Neuroscience. 2011;184:172-82. https://doi.org/10.1016/j.neuroscience.2011.02.057.

36. Ji Y, Tang B, Traub RJ. Spinal estrogen receptor alpha mediates estradiolinduced pronociception in a visceral pain model in the rat. Pain. 2011 152(5):1182-91. https://doi.org/10.1016/j.pain.2011.01.046.
37. Khomula EV, Ferrari LF, Araldi D. Sexual Dimorphism in a Reciprocal Interaction of Ryanodine and IP3 Receptors in the Induction of Hyperalgesic Priming. 2017;37(8):2032-44. https://doi.org/10.1523/jneurosci.2911-16.2017.

38. Ferrari LF, Araldi D, Levine JD. Regulation of Expression of Hyperalgesic Priming by Estrogen Receptor alpha in the Rat. J Pain. 2017;18(5):574-82. https://doi.org/10.1016/j.jpain.2016.12.017.

39. Blurton-Jones M, Tuszynski MH. Estrogen receptor-beta colocalizes extensively with parvalbumin-labeled inhibitory neurons in the cortex, amygdala, basal forebrain, and hippocampal formation of intact and ovariectomized adult rats. J Comp Neurol. 2002;452(3):276-87. https://doi.org/10.1002/cne.10393.

40. Donner N, Handa RJ. Estrogen receptor beta regulates the expression of tryptophan-hydroxylase 2 mRNA within serotonergic neurons of the rat dorsal raphe nuclei. Neuroscience. 2009;163(2):705-18. https://doi.org/10. 1016/j.neuroscience.2009.06.046.

41. Leventhal L, Brandt MR, Cummons TA, Piesla MJ, Rogers KE, Harris HA. An estrogen receptor-beta agonist is active in models of inflammatory and chemical-induced pain. Eur J Pharmacol. 2006;553(1-3):146-8. https://doi. org/10.1016/j.ejphar.2006.09.033.

42. Gardell LR, Hyldtoft L, Del Tredici AL, Andersen CB, Fairbairn LC, Lund BW, et al. Differential modulation of inflammatory pain by a selective estrogen receptor beta agonist. Eur J Pharmacol. 2008;592(1-3):158-9. https://doi.org/ 10.1016/j.ejphar.2008.06.107.

43. Cao DY, Ji Y, Tang B, Traub RJ. Estrogen receptor beta activation is antinociceptive in a model of visceral pain in the rat. J Pain. 2012;13(7):68594. https://doi.org/10.1016/j.jpain.2012.04.010.

44. Imamov O, Yakimchuk K, Morani A, Schwend T, Wada-Hiraike O, Razumov S, et al. Estrogen receptor beta-deficient female mice develop a bladder phenotype resembling human interstitial cystitis. Proc Natl Acad Sci U S A. 2007;104(23):9806-9. https://doi.org/10.1073/pnas.0703410104.

45. Spooner MF, Robichaud P, Carrier JC, Marchand S. Endogenous pain modulation during the formalin test in estrogen receptor beta knockout mice. Neuroscience. 2007;150(3):675-80. https://doi.org/10.1016/j. neuroscience.2007.09.037.

46. Harrington WR, Sheng S, Barnett DH, Petz LN, Katzenellenbogen JA, Katzenellenbogen BS. Activities of estrogen receptor alpha- and betaselective ligands at diverse estrogen responsive gene sites mediating transactivation or transrepression. Mol Cell Endocrinol. 2003;206(1-2):13-22.

47. Jia M, Dahlman-Wright K, Gustafsson JA. Estrogen receptor alpha and beta in health and disease. Best Pract Res Clin Endocrinol Metab. 2015;29(4):55768. https://doi.org/10.1016/j.beem.2015.04.008.

48. Lindberg MK, Moverare S, Skrtic S, Gao H, Dahlman-Wright K, Gustafsson JA, et al. Estrogen receptor (ER)-beta reduces ERalpha-regulated gene transcription, supporting a "ying yang" relationship between ERalpha and ERbeta in mice. Mol Endocrinol. 2003;17(2):203-8. https://doi.org/10.1210/me.2002-0206.

49. Lu YP, Zeng M, Swaab DF, Ravid R, Zhou JN. Colocalization and alteration of estrogen receptor-alpha and -beta in the hippocampus in Alzheimer's disease. Hum Pathol. 2004;35(3):275-80.

50. Chow SK, Leung KS, Qin L, Wei F, Cheung WH. Callus formation is related to the expression ratios of estrogen receptors-alpha and -beta in ovariectomyinduced osteoporotic fracture healing. Arch Orthop Trauma Surg. 2014; 134(10):1405-16. https://doi.org/10.1007/s00402-014-2070-0.

51. Pedram A, Razandi M, Levin ER. Nature of functional estrogen receptors at the plasma membrane. Mol Endocrinol. 2006;20(9):1996-2009. https://doi. org/10.1210/me.2005-0525.

52. Meitzen J, Mermelstein PG. Estrogen receptors stimulate brain region specific metabotropic glutamate receptors to rapidly initiate signal transduction pathways. J Chem Neuroanat. 2011;42(4):236-41. https://doi. org/10.1016/j.jchemneu.2011.02.002.

53. Liu F, Day M, Muniz LC, Bitran D, Arias R, Revilla-Sanchez R, et al. Activation of estrogen receptor-beta regulates hippocampal synaptic plasticity and improves memory. Nat Neurosci. 2008;11(3):334-43. https://doi.org/10.1038/nn2057.

54. Boulware MI, Weick JP, Becklund BR, Kuo SP, Groth RD, Mermelstein PG. Estradiol activates group I and II metabotropic glutamate receptor signaling, leading to opposing influences on CAMP response element-binding protein. J Neurosci. 2005;25(20):5066-78. https://doi.org/10.1523/jneurosci.1427-05.2005.

\section{Publisher's Note}

Springer Nature remains neutral with regard to jurisdictional claims in published maps and institutional affiliations. 